\title{
Cyclic Proofs for Linear Temporal Logic
}

\author{
Ioannis Kokkinis Thomas Studer
}

\begin{abstract}
Annotated sequents provide an elegant approach for the design of deductive systems for temporal logics. Their proof theory, however, is notoriously difficult. It is not even clear how to syntactically show the admissibility of weakening. In this paper, we establish weakening by purely proof-theoretic methods, thus solving an open problem by Brünnler and Lange. We also investigate the role of cut in annotated sequent systems.
\end{abstract}

\section{Introduction}

The proof theory of temporal logics, and of modal fixed point logics in general, is notoriously difficult. It is not even clear how to design a finitary deductive system for linear time temporal logic LTL with nice proof-theoretic properties. In this context, deductive systems featuring infinite long proof branches (together with a global soundness condition) and their cyclic variants have recently obtained much attention, see, for instance, [1, 2, 3, 6, 7, 8].

Brünnler and Lange [2] proposed an elegant formalism with cyclic proofs for LTL using focus games from Lange and Stirling [5] as an inspiration. The main technical feature of their system are annotated sequents, which are employed to derive greatest fixed points. However, some very basic prooftheoretic problems turn out to be surprisingly hard in this setting. For instance, despite the admissibility of several structural rules, including cut, being proved semantically in [2], it remains to prove the same facts by proper proof-theoretic methods. Even the admissibility of weakening, which is quite trivial for most types of sequent calculi, is far from being simple for annotated sequents due to the presence of sequent contexts in the annotations.

As Brünnler and Lange point out [2], the problem with weakening is to be expected since the fact that a certain statement is provable by induction does not imply that a weaker statement is also provable by induction. 
In this paper we provide a solution to this open problem and establish the admissibility of weakening by proof-theoretic means.

Moreover, we present a series of examples that explain the design of Brünnler and Lange's system. Their system is based on annotations that are sets of sets of formulas and it uses several rules to unfold greatest fixed points. Our examples show that these features are necessary in order to have completeness, that means their system is as simple as a cut-free system can be.

However, we also show that if we add a cut-rule, then the system can be made much simpler. That is, we can have fewer rules and annotations of a simpler form. This provides a very nice and instructive example on the role of cut in proofs of induction statements.

\section{Sequent Systems for $L T L$}

In this section we see an approach for defining a sound and complete sequent calculus for LTL. Note that we only study the unary fragment of LTL. This is enough to discuss the proof-theoretic problems but simplifies the presentation.

We start with defining the syntax and semantics for LTL-sequents. Then we recall a naive way of giving an LTL-sequent calculus and explain its shortcomings. We finish this section with introducing the sequent calculus LT1, which is based on so-called annotations.

\subsection{Syntax and Semantics}

We start with a countable set of atomic propositions, which we call Prop. The language of the sequent calculus, $\mathcal{L}_{\mathcal{S}}$, is then described by the following grammar:

$$
A::=P|\bar{P}| A \wedge A|A \vee A| \square A|\diamond A| \bigcirc A
$$

where $P \in$ Prop and $\bar{P}$ denotes the negation of $P$. In this paper, we assume right associativity for all binary connectives.

We define the set of sequents, Seq, by:

$$
\text { Seq }:=\left\{\Gamma \mid \Gamma \text { is a finite subset of } \mathcal{L}_{\mathcal{S}}\right\}
$$

We will use capital greek letters like $\Gamma, \Delta, \Sigma, \ldots$ for sequents, capital latin letters like $A, B, C, D, \ldots$ for $\mathcal{L}_{\mathcal{S}}$-formulas and the letters $P, Q$ for elements 
of Prop, all of them possibly primed or with subscripts. As usual union is represented by comma, i.e.:

$$
\begin{aligned}
& \Gamma, \Delta \text { stands for } \Gamma \cup \Delta \\
& \Gamma, A \text { stands for } \Gamma \cup\{A\}
\end{aligned}
$$

We define the negation of an $\mathcal{L}_{\mathcal{S}}$-formula as usual by:

$$
\begin{array}{rlrl}
\overline{\bar{P}} & :=P & & \overline{\bigcirc A}:=\bigcirc \bar{A} \\
\overline{A \vee B} & :=\bar{A} \wedge \bar{B} & & \overline{\square A}:=\diamond \bar{A} \\
\overline{A \wedge B}:=\bar{A} \vee \bar{B} & & \overline{\nabla A}:=\square \bar{A}
\end{array}
$$

Let $\Gamma \in$ Seq. We define the following sequent:

$$
\bigcirc \Gamma:=\{\bigcirc A \mid A \in \Gamma\}
$$

Now we can define the notion of LTL-model and validity.

Definition 1 (LTL-model). An LTL-model or simply a model is a function $\mu$ that maps natural numbers to sets of atomic propositions, i.e.: ${ }^{1}$

$$
\mu: \mathbb{N} \rightarrow \mathcal{P}(\text { Prop })
$$

Let $\mu$ be a model. Every natural number $i$ represents a point in time and the set $\mu(i)$ represents the facts that hold at the time-point $i$. The expression $\mu, i \models A$ stands for model $\mu$ satisfies formula $A$ at time-point $i$. The relation $\models$ is defined as follows:

Definition 2 (Satisfiability of $\mathcal{L}_{\mathcal{S}^{-}}$-formulas). Let $\mu$ be a model and let $i \in \mathbb{N}$. We have:

$$
\begin{aligned}
\mu, i \models P & \Longleftrightarrow P \in \mu(i) \\
\mu, i \models \bar{P} & \Longleftrightarrow P \notin \mu(i) \\
\mu, i \models A \wedge B & \Longleftrightarrow \mu, i \models A \text { and } \mu, i \models B \\
\mu, i \models A \vee B & \Longleftrightarrow \mu, i \models A \text { or } \mu, i \models B \\
\mu, i \models \square A & \Longleftrightarrow \forall j \geq i(\mu, j \models A) \\
\mu, i \models \diamond A & \Longleftrightarrow \exists j \geq i(\mu, j \models A) \\
\mu, i \models \bigcirc A & \Longleftrightarrow \mu, i+1 \models A
\end{aligned}
$$

\footnotetext{
${ }^{1} \mathcal{P}$ stands for powerset
} 
A formula $A$ is valid, denoted by $\models A$, iff $(\forall \mu)(\forall i)[\mu, i \models A]$, i.e. iff $A$ holds in all models at all time-points.

Let $\Gamma \in$ Seq. We assume that $\Gamma$ is semantically equivalent to the disjunction of its elements, i.e. we set

$$
\mu, i \models \Gamma \quad \Longleftrightarrow \quad \mu, i \models \bigvee_{A \in \Gamma} A
$$

\subsection{A Naive Approach}

We begin with a "naive" sequent calculus for LTL, called $L T_{\text {naive, }}$ which is given in Figure 1. This system contains the usual propositional axioms and rules (aid, $\vee, \wedge$ ), the rule for $\bigcirc$ and the rules for unfolding $\square$ and $\diamond$.

As usual $\mathrm{L} \vdash A$ means that the logic $\mathrm{L}$ proves the formula $A$. When $\mathrm{L}$ is clear from the context we may simply write $\vdash A$. By $\mathrm{L} \vdash^{n} A$ we mean that there is a derivation of $A$ in $\mathrm{L}$ with depth at most $n$.

$$
\begin{gathered}
\text { aid } \frac{}{\Gamma, P, \bar{P}} \vee \frac{\Gamma, A, B}{\Gamma, A \vee B} \wedge \frac{\Gamma, A \quad \Gamma, B}{\Gamma, A \wedge B} \\
\square \frac{\Gamma, A \quad \Gamma, \bigcirc \square A}{\Gamma, \square A} \quad \diamond \frac{\Gamma, A, \bigcirc \diamond A}{\Gamma, \diamond A} \quad \bigcirc \frac{\Gamma}{\Sigma, \bigcirc \Gamma}
\end{gathered}
$$

Figure 1: System LT $_{\text {naive }}$

It is easy to see that system $L T_{\text {naive }}$ is sound with respect to $L T L$-models.

But what about completeness? The first observation, see [2], is that system $\mathrm{LT}_{\text {naive }}$ almost works: the only thing that goes wrong is that we cannot derive induction principles as is shown in the following example.

Example 3. The valid sequent

$$
\Gamma=\diamond(P \wedge \bigcirc \bar{P}), \bar{P}, \square P
$$

cannot be proved in $\mathrm{LT}_{\text {naive }}$. This sequent is semantically equivalent to the temporal induction axiom

$$
P \wedge \square(P \rightarrow \bigcirc P) \rightarrow \square P
$$


where the connective $\rightarrow$ is interpreted in the standard way.

An attempt to prove $\Gamma$ in $L T_{\text {naive }}$ will lead to a derivation like the following:

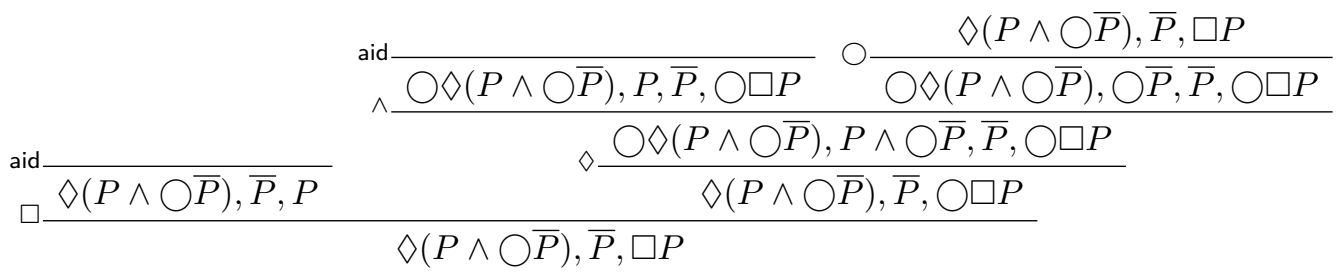

Observe that the endsequent reappears in the top right of the derivation. Hence there is no proof of $\Gamma$ in $\mathrm{LT}_{\text {naive }}$.

When we try to prove a formula that contains the operator $\square$, the proofsearch will fail like it did in the above example for sequent $\Gamma$. However, the obvious idea of just closing a cyclic branch as axiomatic will lead to an unsound system as is illustrated in the next example, see [2].

Example 4. Consider the non-valid sequent $\Delta=\square P, \bigcirc \diamond \square P$. If we could close all the cyclic branches then we would have the following proof for $\Delta$ in $\mathrm{LT}_{\text {naive }}$ :

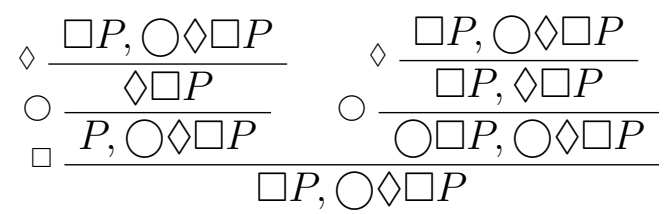

Hence, a better idea, than simply closing every cyclic branch, is required. Brünnler and Lange's idea ([2]) is to close a cyclic branch if there is a formula such that whenever the $\square$-rule is applied to it between the two occurrences of the cyclic sequent, the branch is along the right premise. Thus, in Example 3,

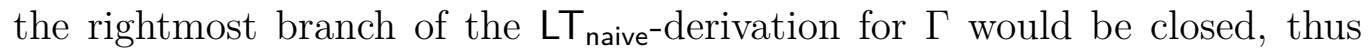
yielding a correct proof. In Example 4, however, the left branch in the derivation for $\Delta$ would not be closed and hence this would not be a proof for the non-valid sequent $\Delta$. In order to implement this idea, we have to enrich our syntax with the so-called annotations.

\subsection{Annotations - System LT1}

We define the set of annotated formulas, $\mathcal{L}_{\text {ann }}$ :

$$
\mathcal{L}_{\text {ann }}:=\left\{\square_{\Gamma} A, \bigcirc \square_{\Gamma} A \mid A \in \mathcal{L}_{\mathcal{S}}, \Gamma \in \text { Seq }\right\}
$$


In $\square_{\Gamma} A$, the sequent $\Gamma$ is called an annotation. We define the set of annotated sequents:

$$
\begin{aligned}
\text { Seq ann }_{\text {an }}:=\left\{\Gamma \mid \Gamma \text { is a finite subset of } \mathcal{L}_{\mathcal{S}} \cup \mathcal{L}_{\text {ann }}\right. \text { that contains } \\
\text { at most one annotated formula }\}
\end{aligned}
$$

The semantics of $\square_{\Gamma} A$ is defined as follows. Let $\mu$ be an LTL-model and let $i \in \mathbb{N}$. We have:

$$
\mu, i \models \square_{\Gamma} A \quad \Longleftrightarrow \quad \forall j \geq i((\forall i \leq k \leq j(\mu, k \models \Gamma)) \Longrightarrow \mu, j \models A)
$$

System LT1 is given by the axioms and rules in Figure 2. For the $\bigcirc$-rule, we assume $\Sigma \in$ Seq, i.e. $\Sigma$ does not contain annotated formulas. For rep and foc, we also assume $\Gamma \in$ Seq.

$$
\begin{gathered}
\text { aid } \frac{}{\Gamma, P, \bar{P}} \quad \vee \frac{\Gamma, A, B}{\Gamma, A \vee B} \quad \wedge \frac{\Gamma, A \quad \Gamma, B}{\Gamma, A \wedge B} \\
\operatorname{rep} \frac{\Gamma, \square_{\Gamma} A}{\Gamma} \quad \text { foc } \frac{\Gamma, A \quad \Gamma, \bigcirc \square_{\Gamma} A}{\Gamma, \square A} \\
\square \frac{\Gamma, A \quad \Gamma, \bigcirc \square A}{\Gamma, \square A} \quad \diamond \frac{\Gamma, A, \bigcirc \diamond A}{\Gamma, \diamond A} \quad \bigcirc \frac{\Gamma}{\Sigma, \bigcirc \Gamma}
\end{gathered}
$$

Figure 2: System LT1

System LT1 contains all the rules of the system $L T_{\text {naive }}$ plus the rule foc and the axiom rep. The name of the rule foc stands for focus and implies that we focus on a specific $\square$-formula, i.e. the formula $\square A$ in the conclusion of the foc-rule. We focus on this formula by annotating it with its context, i.e. $\Gamma$. When the annotated formula appears in exactly the same context (i.e. $\Gamma$ ), then we can close the branch as axiomatic using the axiom rep (the name of this axiom stands for repetition).

The sequent $\diamond(P \wedge \bigcirc \bar{P}), \bar{P}, \square P$ (which could not be proved in system $\mathrm{LT}_{\text {naive }}$ ) can be derived in LT1 as follows: 


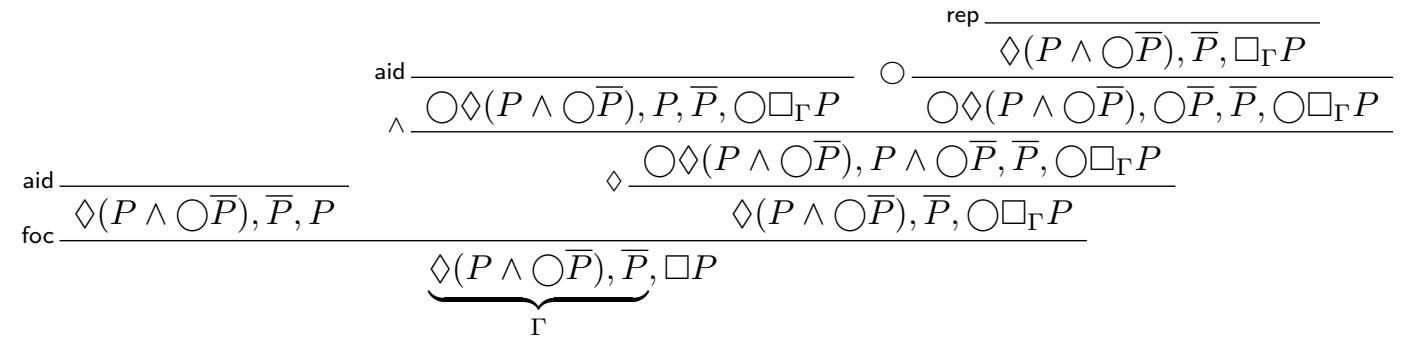

Brünnler and Lange [2] present a soundness proof for a system very similar to LT1. However, as we will see later, system LT1 only is complete if we add a cut rule. We define system LT1 ${ }^{\text {cut }}$ to be LT1 enriched with the rule cut:

$$
\text { cut } \frac{\Gamma, A \quad \bar{A}, \Delta}{\Gamma, \Delta}
$$

In this rule, $A$ is an $\mathcal{L}_{\mathcal{S}}$-formula.

Before showing the completeness and incompleteness results we need to show that weakening is syntactically admissible in system LT1 ${ }^{\text {cut }}$.

Definition 5. A derivation satisfies the next-property iff any branch from the endsequent to any instance of foc goes through at least one $\bigcirc$-rule.

Lemma 6. Let $\Gamma \in$ Seq. If $\mathrm{LT} 1^{\text {cut }} \mapsto^{n} \Gamma$, then there is an $\mathrm{LT} 1^{\text {cut }}$-proof of $\Gamma$ satisfying the next-property.

Proof. By induction on $n$ and a case distinction on the last rule.

1. $\Gamma$ is the conclusion of aid. Then the claim holds trivially.

2. $\Gamma$ is the conclusion of rep. This case is not possible because of our assumption $\Gamma \in$ Seq.

3. $\Gamma$ is the conclusion of foc. Then $\Gamma=\Delta, \square A$ and the given proof $\mathcal{E}$ of $\Gamma$ has the following form:

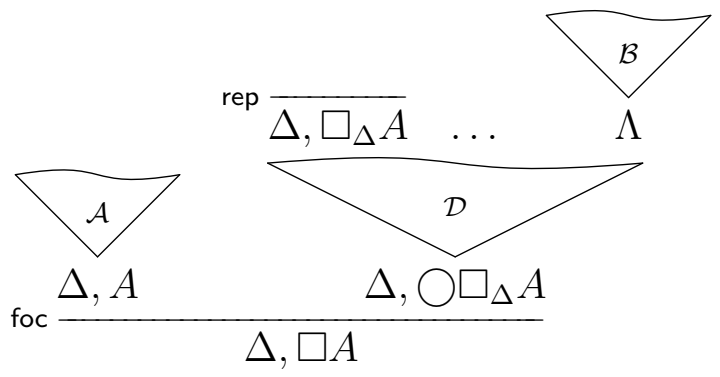


In the above derivation the sequent $\Lambda$ contains no annotations, i.e. $\Lambda \in$ Seq. All the other sequents in derivation $\mathcal{D}$ contain annotations. Obviously $\Lambda$ is obtained by an application of the rule cut.

First we observe that by the induction hypothesis, there are proofs $\mathcal{A}^{\prime}$ and $\mathcal{B}^{\prime}$ of $\Delta, A$ and $\Lambda$ respectively that satisfy the next-property.

Further we have that in the derivation $\mathcal{D}$

$$
\begin{aligned}
& \text { any branch from } \Delta, \bigcirc \square_{\Delta} A \text { to some } \Delta, \square_{\Delta} A \\
& \text { goes through a } \bigcirc \text {-rule }
\end{aligned}
$$

and

$$
\text { there are no instances of foc. }
$$

Let $\mathcal{D}^{\prime}$ be the derivation that is obtained from $\mathcal{D}$ by dropping all annotations occurring in threads starting from $\bigcirc \square_{\Delta} A$, i.e. by replacing sequents of the form $\Sigma, \square_{\Delta} A$ and $\Sigma, \bigcirc \square_{\Delta} A$ by $\Sigma, \square A$ and $\Sigma, \bigcirc \square A$, rescpectively.

Hence we have the following proof of $\Delta, \square A$ :

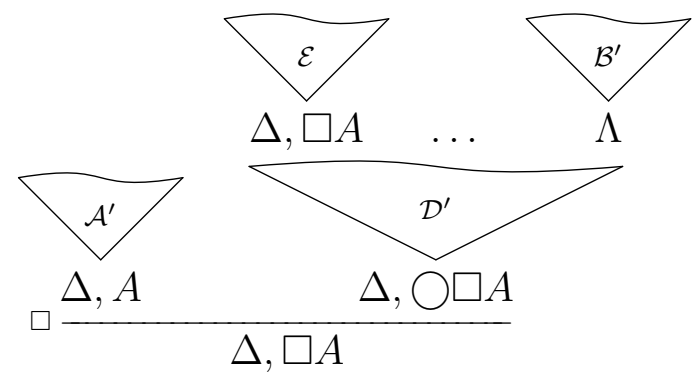

This proof satisfies the next-property. Indeed:

(a) The proofs $\mathcal{A}^{\prime}$ and $\mathcal{B}^{\prime}$ satisfy the next-property.

(b) In the derivation $\mathcal{D}^{\prime}$ every branch from $\Delta, \bigcirc \square A$ to some $\Delta, \square A$ goes through at least one $\bigcirc$-rule (because of (1)).

(c) The derivation $\mathcal{D}^{\prime}$ contains no foc-rules (because of (2)).

Hence any branch from the conclusion $\Delta, \square A$ to an instance of foc goes through a $\bigcirc$-rule.

4. In all other cases, the claim follows easily by the induction hypothesis.

By Lemma 6 we immediately get the following weakening result. 
Corollary 7 (Weakening for non-annotated sequents). For any $\Gamma, \Delta \in$ Seq we have:

$$
\mathrm{LT}^{\text {cut }} \vdash \Gamma \Longrightarrow \mathrm{LT} 1^{\text {cut }} \vdash \Gamma, \Delta
$$

Proof. Let $\mathrm{LT} 1^{\text {cut }} \vdash \Gamma$. By Lemma 6 we have a proof $\mathcal{D}$ for $\Gamma$ that satisfies the next-property. We prove the claim by induction on the length of $\mathcal{D}$. Let $\alpha$ be the lowermost rule in $\mathcal{D}$. Since $\mathcal{D}$ satisfies the next-property and $\Gamma \in \mathrm{Seq}$, the rule $\alpha$ can be aid, $\vee, \wedge, \square, \diamond$, cut or $\bigcirc$. If $\alpha$ is aid or $\bigcirc$, then claim follows by built-in weaking. Otherwise it follows by the induction hypothesis.

By a similar proof, we can show invertibiltiy of $\vee$.

Corollary 8 (Invertibility of the $\vee$-rule). For any $\Gamma \in \operatorname{Seq}$ and $A, B \in \mathcal{L}_{\mathcal{S}}$ we have:

$$
\mathrm{LT}^{\text {cut }} \vdash \Gamma, A \vee B \Longrightarrow \mathrm{LT}^{\text {cut }} \vdash \Gamma, A, B
$$

\section{Completeness}

In this section, we show that system $\mathrm{LT} 1^{\text {cut }}$ is complete by embedding a complete Hilbert system for LTL in $L T 1^{\text {cut }}$. We will also show that the cutfree system LT1 is not complete and that we need more complex annotations to obtain a complete cut-free system.

\subsection{A Hilbert System for LTL}

The language $\mathcal{L}_{\mathcal{H}}$ is described by the following grammar:

$$
\phi:=P|\neg \phi| \phi \wedge \phi|\square \phi| \bigcirc \phi
$$

where $P \in$ Prop. Additionally we will use the following abbreviations:

$$
\begin{array}{rlrl}
\phi \vee \psi & :=\neg(\neg \phi \wedge \neg \psi) & \diamond \phi & :=\neg \square \neg \phi \\
\phi \rightarrow \psi & :=\neg(\phi \wedge \neg \psi) & \phi \leftrightarrow \psi:=(\phi \rightarrow \psi) \wedge(\psi \rightarrow \phi)
\end{array}
$$

We will use the Greek letters $\phi, \psi, \rho, \ldots$ for $\mathcal{L}_{\mathcal{H}}$-formulas possibly primed or with subscripts.

Figure 3 shows the Hilbert system $\Sigma_{\text {LTL }}$ for LTL.

Satisfiability of $\mathcal{L}_{\mathcal{H}}$-formulas in an LTL-model is defined as follows: 


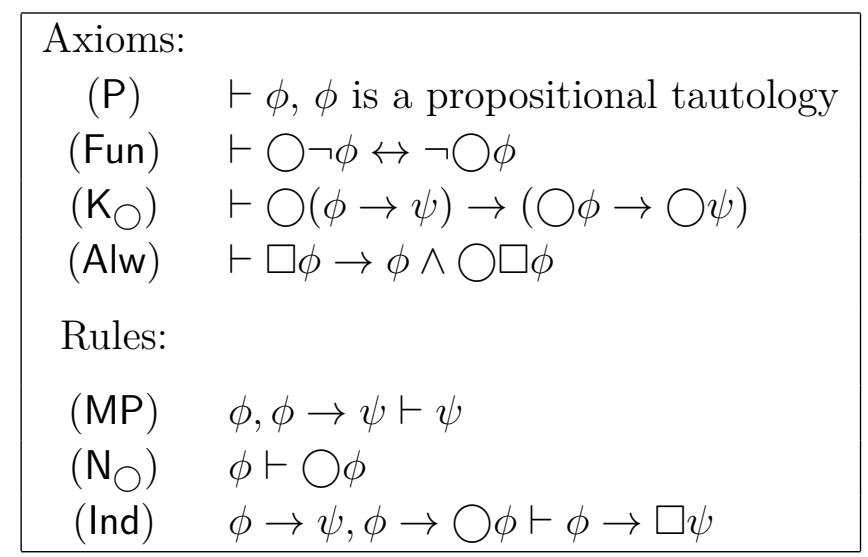

Figure 3: System $\Sigma_{\text {LTL }}$

Definition 9 (Satisfiability of $\mathcal{L}_{\mathcal{H}}$-formulas). Let $\mu$ be a model and $i \in \mathbb{N}$. We have:

$$
\begin{aligned}
\mu, i \models P & \Longleftrightarrow P \in \mu(i) \\
\mu, i \models \neg \phi & \Longleftrightarrow \mu, i \models \phi \\
\mu, i \models \phi \wedge \psi & \Longleftrightarrow \mu, i \models \phi \text { and } \mu, i \models \psi \\
\mu, i \models \square \phi & \Longleftrightarrow \forall j \geq i(\mu, j \models \phi) \\
\mu, i \models \bigcirc \phi & \Longleftrightarrow \mu, i+1 \models \phi
\end{aligned}
$$

Validity for $\mathcal{L}_{\mathcal{H}}$-formulas is defined in the same way as for $\mathcal{L}_{\mathcal{S}}$-formulas. The following theorem is proved in Chapter 2 of [4].

Theorem 10 (Soundness and Completeness). The system $\Sigma_{\mathrm{LTL}}$ is sound and complete with respect to LTL-models, i.e. we have for all $\mathcal{L}_{\mathcal{H}}$-formulas $\phi$ :

$$
\models \phi \quad \Longleftrightarrow \quad \Sigma_{\mathrm{LTL}} \vdash \phi
$$

\subsection{System LT1 + cut is Complete}

In this subsection we will show that the system $\mathrm{LT} 1^{\text {cut }}$ is complete by embedding system $\Sigma_{\text {LTL }}$ to system $L T 1^{\text {cut }}$. Before proving the embedding we need some auxiliary definitions and lemmata.

As a first step we extend axiom aid to all $\mathcal{L}_{\mathcal{S}}$-formulas.

Lemma 11. [Extension of axiom aid to $\mathcal{L}_{\mathcal{S}}$-formulas] Let $\Gamma \in$ Seq and let $A \in \mathcal{L}_{\mathcal{S}}$. Then we have the following in system $\mathrm{LT}^{\text {cut }}$ :

$$
\vdash \Gamma, A, \bar{A}
$$


Proof. The proof is by induction on the structure of $A$. The only interesting cases are $A=\square B$ and $A=\diamond B$. So assume $A=\square B$. It holds $\bar{A}=\diamond \bar{B}$. Then we have the following derivation in $\mathrm{LT} 1^{\text {cut }}$ :

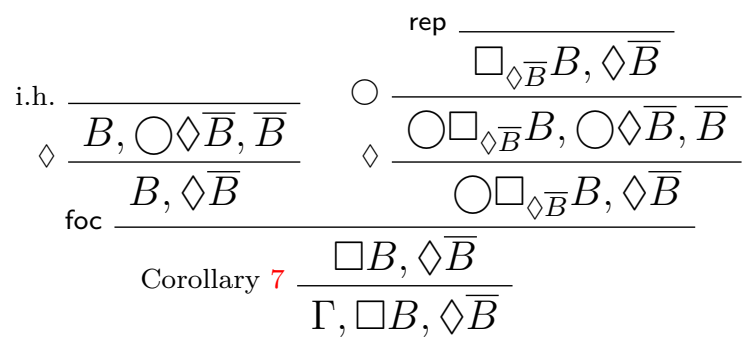

The case $A=\diamond B$ simply is dual to the shown case.

An easy induction on the structure of the formula $A$ also yields the following lemma.

Lemma 12. Let $A \in \mathcal{L}_{\mathcal{S}}$. It holds that:

$$
\overline{\bar{A}}=A
$$

Now we define two translation functions between the languages $\mathcal{L}_{\mathcal{H}}$ and $\mathcal{L}_{\mathcal{S}}$.

We define the function $\sigma: \mathcal{L}_{\mathcal{S}} \rightarrow \mathcal{L}_{\mathcal{H}}$ inductively:

$$
\begin{array}{rlrl}
\sigma(P) & =P & \sigma(\bar{P}) & =\neg P \\
\sigma(A \wedge B) & =\sigma(A) \wedge \sigma(B) & \sigma(A \vee B) & =\sigma(A) \vee \sigma(B) \\
\sigma(\square A) & =\square \sigma(A) & \sigma(\diamond A) & =\diamond \sigma(A) \\
\sigma(\bigcirc A) & =\bigcirc \sigma(A) &
\end{array}
$$

We define the function $\tau: \mathcal{L}_{\mathcal{H}} \rightarrow \mathcal{L}_{\mathcal{S}}$ inductively:

$$
\begin{aligned}
\tau(P) & =P & \tau(\square \phi) & =\square \tau(\phi) \\
\tau(\neg \phi) & =\overline{\tau(\phi)} & \tau(\bigcirc \phi) & =\bigcirc \tau(\phi) \\
\tau(\phi \wedge \psi) & =\tau(\phi) \wedge \tau(\psi) & &
\end{aligned}
$$

Some simple calculations show that the function $\tau$ behaves as expected with respect to the propositional connectives.

Lemma 13. Let $\phi, \psi \in \mathcal{L}_{\mathcal{H}}$. It holds:

$$
\text { (1) } \tau(\phi \rightarrow \psi)=\overline{\tau(\phi)} \vee \tau(\psi)
$$


(2) $\tau(\phi \vee \psi)=\tau(\phi) \vee \tau(\psi)$

(3) $\tau(\phi \leftrightarrow \psi)=(\overline{\tau(\phi)} \vee \tau(\psi)) \wedge(\overline{\tau(\psi)} \vee \tau(\phi))$

(4) $\tau(\diamond \phi)=\diamond \tau(\phi)$

It is straightforward to show that $\tau$ is the inverse of $\sigma$.

Lemma 14. Let $A \in \mathcal{L}_{\mathcal{S}}$. It holds:

$$
\tau(\sigma(A))=A
$$

Now we can prove the embedding Lemma:

Lemma 15 (Embedding of $\Sigma_{\text {LTL into LT1 }} 1^{\text {cut }}$ ).

$$
\left(\forall \phi \in \mathcal{L}_{\mathcal{H}}\right)\left[\Sigma_{\mathrm{LTL}} \vdash \phi \Longrightarrow \mathrm{LT} 1^{\text {cut }} \vdash \tau(\phi)\right]
$$

Proof. By induction on the length of the derivation $\Sigma_{\mathrm{LTL}} \vdash \phi$. We distinguish the following cases:

1. $\phi$ is a propositional tautology. Then $\tau(\phi)$ is also a propositional tautology. Hence, clearly LT1 ${ }^{\text {cut }} \vdash \tau(\phi)$.

2. $\phi$ is an instance of the axiom (Fun). That means there is a $\psi \in \mathcal{L}_{\mathcal{H}}$ such that $\phi=\neg \bigcirc \psi \leftrightarrow \bigcirc \neg \psi$. From Lemma 12 and Lemma 13 we get:

$$
\tau(\phi)=(\bigcirc \tau(\psi) \vee \bigcirc \overline{\tau(\psi)}) \wedge(\bigcirc \tau(\psi) \vee \bigcirc \overline{\tau(\psi)})
$$

So, we have the following derivation in $\mathrm{LT} 1^{\text {cut }}$ :

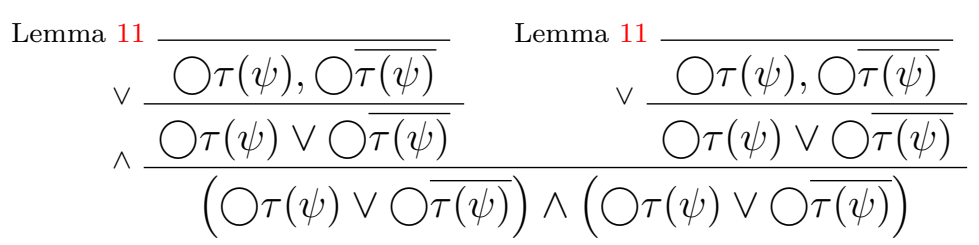

3. $\phi$ is an instance of axiom $\left(\mathrm{K}_{\bigcirc}\right)$. That means there are $\psi, \rho \in \mathcal{L}_{\mathcal{H}}$ such that $\phi=\bigcirc(\psi \rightarrow \rho) \rightarrow \bigcirc \psi \rightarrow \bigcirc \rho$. By Lemma 12 and Lemma 13 we get:

$$
\tau(\phi)=\bigcirc(\tau(\psi) \wedge \overline{\tau(\rho)}) \vee \bigcirc \overline{\tau(\psi)} \vee \bigcirc \tau(\rho)
$$

So, we have the following derivation: 


$$
\begin{aligned}
\wedge & \frac{\overline{\tau(\psi), \overline{\tau(\psi)}, \tau(\rho)} \quad \text { Lemma } 11 \overline{\overline{\tau(\rho)}}, \overline{\tau(\psi)}, \tau(\rho)}{} \\
& \vee \frac{\bigcirc(\psi) \wedge \overline{\tau(\rho)}, \overline{\tau(\psi)}, \tau(\rho)}{\bigcirc(\tau(\psi) \wedge \overline{\tau(\rho)}), \bigcirc \overline{\tau(\psi)}, \bigcirc \tau(\rho)} \\
& \vee \frac{\bigcirc(\tau(\psi) \wedge \overline{\tau(\rho)}) \vee \bigcirc \overline{\tau(\psi)}, \bigcirc \tau(\rho)}{\bigcirc(\tau(\psi) \wedge \overline{\tau(\rho)}) \vee \bigcirc \overline{\tau(\psi)} \vee \bigcirc \tau(\rho)}
\end{aligned}
$$

4. $\phi$ is an instance of the axiom (Alw). That means there is a $\psi \in \mathcal{L}_{\mathcal{H}}$ such that $\phi=\square \psi \rightarrow \psi \wedge \bigcirc \square \psi$. From Lemma 12 and Lemma 13 we get:

$$
\tau(\phi)=\diamond \overline{\tau(\psi)} \vee(\tau(\psi) \wedge \bigcirc \square \tau(\psi))
$$

So, we have the following derivation in $\mathrm{LT} 1^{\text {cut }}$ :

$$
\begin{aligned}
\wedge \frac{\overline{\tau(\psi)}, \bigcirc \diamond \overline{\tau(\psi)}, \tau(\psi)}{\bar{\tau}} \quad \text { Lemma } 11 \overline{\overline{\tau(\psi)}, \bigcirc \diamond \overline{\tau(\psi)}, \bigcirc \square \tau(\psi)} \\
\qquad \frac{\overline{\tau(\psi)}, \bigcirc \diamond \overline{\tau(\psi)}, \tau(\psi) \wedge \bigcirc \square \tau(\psi)}{\vee \frac{\diamond \overline{\tau(\psi)}, \tau(\psi) \wedge \bigcirc \square \tau(\psi)}{\diamond \overline{\tau(\psi)} \vee(\tau(\psi) \wedge \bigcirc \square \tau(\psi))}}
\end{aligned}
$$

5. $\phi$ is the conclusion of an application of (MP). That means there is a $\psi \in \mathcal{L}_{\mathcal{H}}$ such that $\Sigma_{\mathrm{LTL}} \vdash \psi$ and $\Sigma_{\mathrm{LTL}} \vdash \psi \rightarrow \phi$. Then we find the following derivation in $\mathrm{LT} 1^{\text {cut }}$ :

$$
\begin{array}{lr}
\text { Lemma } 13(1) \frac{\text { i.h. }}{\overline{\tau(\psi \rightarrow \phi)}} \\
\text { cut } \frac{\overline{\tau(\psi)} \vee \tau(\phi)}{\tau(\psi)} \\
\text { Corollary } 8 \frac{\overline{\tau(\psi)}, \tau(\phi)}{\tau(\phi)}
\end{array}
$$

6. $\phi$ is the conclusion of an application of $\left(\mathrm{N}_{\bigcirc}\right)$. That means that there is a $\psi \in \mathcal{L}_{\mathcal{H}}$ such that $\phi=\bigcirc \psi$ and that $\Sigma_{\mathrm{LTL}} \vdash \psi$. In $\mathrm{LT}^{\text {cut }}$ we have the following derivation:

$$
\text { i.h. } \frac{}{\tau(\psi)}
$$

And since $\bigcirc \tau(\psi)=\tau(\bigcirc \psi)=\tau(\phi)$ we have that $\mathrm{LT}^{\text {cut }} \vdash \tau(\phi)$. 
7. $\phi$ is the conclusion of an application of (Ind). That means there are $\psi, \rho \in \mathcal{L}_{\mathcal{H}}$ such that $\phi=\psi \rightarrow \square \rho$, and that $\Sigma_{\mathrm{LTL}} \vdash \psi \rightarrow \rho$ and $\Sigma_{\mathrm{LTL}} \vdash \psi \rightarrow \bigcirc \psi$. By Lemma 13 (1) we have:

$$
\begin{aligned}
\tau(\phi) & =\tau(\psi \rightarrow \square \rho)=\overline{\tau(\psi)} \vee \square \tau(\rho) \\
\tau(\psi \rightarrow \rho) & =\overline{\tau(\psi)} \vee \tau(\rho) \\
\tau(\psi \rightarrow \bigcirc \psi) & =\overline{\tau(\psi)} \vee \bigcirc \tau(\psi)
\end{aligned}
$$

We find the following derivation in $\mathrm{LT} 1^{\text {cut }}$ :

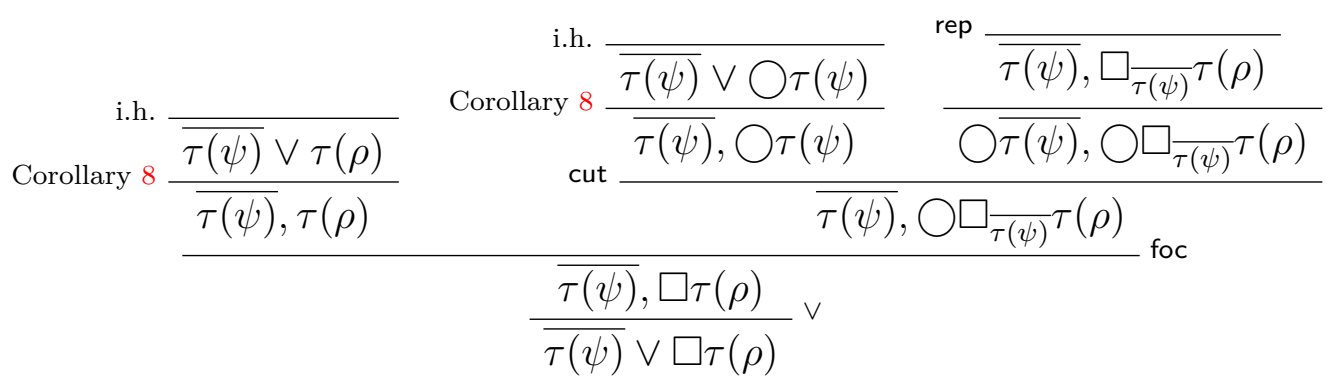

To establish completeness of LT1 ${ }^{\text {cut }}$, we need the following lemma, which can easily be shown by induction on $A$.

Lemma 16. Let $A \in \mathcal{L}_{\mathcal{S}}$, let $\mu$ be a model and let $i \in \mathbb{N}$. It holds:

$$
\mu, i \models A \quad \Longrightarrow \quad \mu, i \models \sigma(A)
$$

Finally we can prove completeness of system $\mathrm{LT} 1^{\text {cut }}$.

Theorem 17. System $\mathrm{LT} 1^{\text {cut }}$ is complete for $\mathcal{L}_{\mathcal{S}}$-formulas, i.e. for all $\mathcal{L}_{\mathcal{S}^{-}}$ formulas $A$

$$
\models A \quad \Longrightarrow \quad \mathrm{LT}^{\mathrm{cut}} \vdash A
$$

Proof. Let $A \in \mathcal{L}_{\mathcal{S}}$. We have:

$$
\begin{aligned}
& \models A \Longrightarrow(\forall \mu)(\forall i \in \mathbb{N})[\mu, i \models A] \stackrel{\text { Lemma }}{\Longrightarrow} 16 \\
& (\forall \mu)(\forall i \in \mathbb{N})[\mu, i \models \sigma(A)] \Longrightarrow \models \sigma(A) \stackrel{\text { Theorem }}{\Longrightarrow} 10 \\
& \Sigma_{\text {LTL }} \vdash \sigma(A) \stackrel{\text { Lemma }^{15}}{\Longrightarrow} \mathrm{LT}^{\text {cut }} \vdash \tau(\sigma(A)) \stackrel{\text { Lemma }^{14}}{\Longrightarrow} \mathrm{LT}^{\text {cut }} \vdash A
\end{aligned}
$$




\subsection{System LT1 is not Complete}

Now we show that if we remove the rule cut from system $L T 1^{\text {cut }}$, the resulting system (i.e. LT1) is not complete.

Let $\Gamma$ be the following valid sequent:

$$
\bar{P}, \diamond(P \wedge \bigcirc \bigcirc \bar{P}), \square(P \vee \bigcirc P)
$$

$\Gamma$ is semantically equivalent to the following $\mathcal{L}_{\mathcal{H}}$-formula:

$$
P \wedge \square(P \rightarrow \bigcirc \bigcirc P) \rightarrow \square(P \vee \bigcirc P)
$$

which expresses a valid induction statement in LTL.

The following derivation is an attempt to prove $\Gamma$ in LT1.

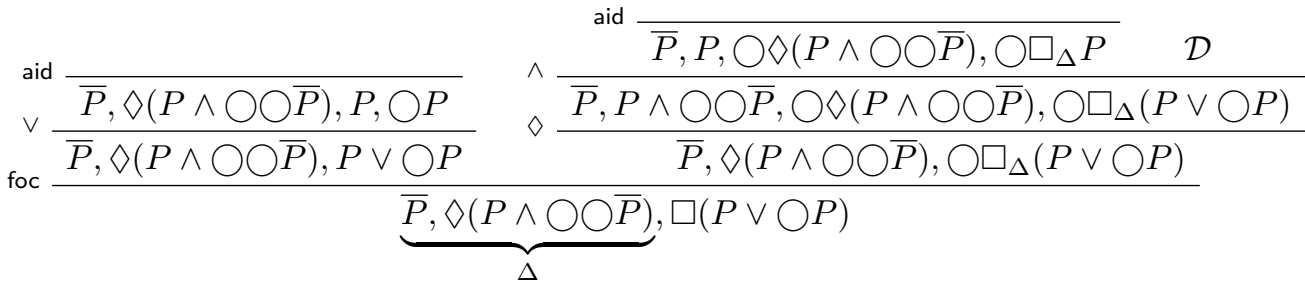

$$
\begin{aligned}
& \mathcal{D}\left\{\bigcirc \frac{\bigcirc \bar{P}, \diamond(P \wedge \bigcirc \bigcirc \bar{P}), \square_{\Delta}(P \vee \bigcirc P)}{\bar{P}, \bigcirc \bigcirc \bar{P}, \bigcirc \diamond(P \wedge \bigcirc \bigcirc \bar{P}), \bigcirc \square_{\Delta}(P \vee \bigcirc P)}\right.
\end{aligned}
$$

The reason we cannot prove sequent $\Gamma$ in system LT1 is that in system LT1 it is impossible to "get rid of an annotation". So, in the above proof-attempt for $\Gamma$ there is no way we can drop $\Delta$ from sequent $\bigcirc \bar{P}, \diamond(P \wedge \bigcirc \bigcirc \bar{P}), \square_{\Delta}(P \vee \bigcirc P)$, which is the sequent on the top of the derivation $\mathcal{D}$. So, the only way to prove an annotated sequent is to reach either axiom rep or axiom aid from it. In our case it is impossible to reach rep from $\bigcirc \bar{P}, \diamond(P \wedge \bigcirc \bigcirc \bar{P}), \square_{\Delta}(P \vee \bigcirc P)$, since it would require the application of a $\bigcirc$-rule, which is not possible (the conclusion of a $\bigcirc$-rule cannot contain a formula of the form $\square_{\Delta} A$ ). It is also impossible to reach aid from $\bigcirc \bar{P}, \diamond(P \wedge \bigcirc \bigcirc \bar{P}), \square_{\Delta}(P \vee \bigcirc P)$ since the sequent $\bigcirc \bar{P}, \diamond(P \wedge \bigcirc \bigcirc \bar{P})$ is not valid and our system is sound. Thus, proof-search for $\Gamma$ in LT1 fails and, therefore, system LT1 is not complete.

We can tackle the problem of "being unable to get rid of annotations" by introducing a new rule. For any $\Delta \in$ Seq we define the following rule:

$$
\square_{\Delta} \frac{\Gamma, A \quad \Gamma, \bigcirc \square_{\Delta} A}{\Gamma, \square_{\Delta} A}
$$


Rule $\square_{\Delta}$ is sound with respect to LTL-models for any $\Delta$. As we can see, rule $\square_{\Delta}$ allows us to drop the annotation from an annotated sequent (the left premise of the rule $\square_{\Delta}$ is an unannotated sequent). We define $\mathrm{LT}^{+}$to be system LT1 plus the rule $\square_{\Delta}$ for any $\Delta \in$ Seq.

In system $\mathrm{LT}^{+}{ }^{+}$we can prove the sequent $\bar{P}, \diamond(P \wedge \bigcirc \bigcirc \bar{P}), \square(P \vee \bigcirc P)$ as follows:

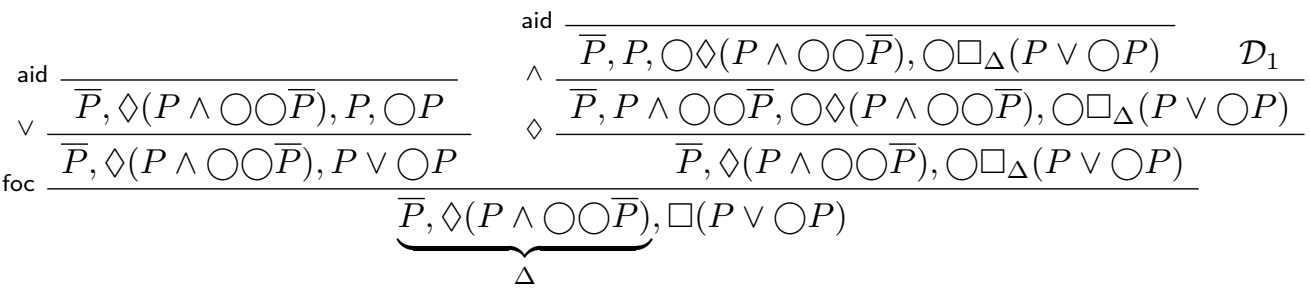

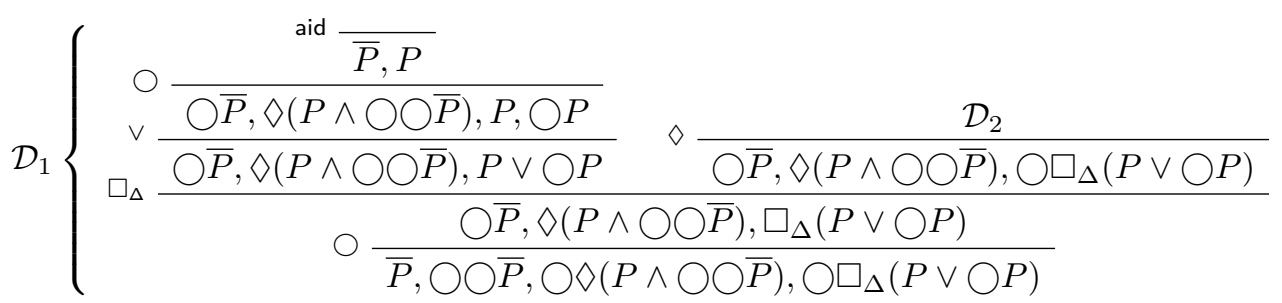

$$
\begin{aligned}
& \mathcal{D}_{2}\left\{\begin{array}{l}
\bigcirc \frac{\mathrm{rep}}{\bar{P}, \diamond(P \wedge \bigcirc \bigcirc \bar{P}), \square_{\Delta}(P \vee \bigcirc P)} \\
\wedge \frac{\bigcirc \bar{P}, P, \bigcirc \diamond(P \wedge \bigcirc \bigcirc \bar{P}), \bigcirc \square_{\Delta}(P \vee \bigcirc P)}{\bigcirc \bar{P}, P \wedge \bigcirc \bigcirc \bar{P}, \bigcirc \diamond(P \wedge \bigcirc \bigcirc \bar{P}), \bigcirc \square_{\Delta}(P \vee \bigcirc P)} \mathcal{D}_{3}
\end{array}\right. \\
& \mathcal{D}_{3}\left\{\bigcirc \frac{\text { rep } \overline{\bar{P}, \diamond(P \wedge \bigcirc \bigcirc \bar{P}), \square_{\Delta}(P \vee \bigcirc P)}}{\bigcirc \bar{P}, \bigcirc \bigcirc \bar{P}, \bigcirc \diamond(P \wedge \bigcirc \bigcirc \bar{P}), \bigcirc \square_{\Delta}(P \vee \bigcirc P)}\right.
\end{aligned}
$$

However, $\mathrm{LT}^{+}$is still too simple: it fails to prove all valid sequents. Take for example the following valid sequent, which we call $\Sigma$ :

$$
\bigcirc \square(\bar{P} \vee \bar{Q}), \diamond(\bigcirc P \wedge \bigcirc Q)
$$

where $P, Q$ are different elements of Prop. We show that $\Sigma$ cannot be derived in $\mathrm{LT} 1^{+}$.

We set $C=\bar{P} \vee \bar{Q}$ and $D=\bigcirc P \wedge \bigcirc Q$. A proof-attempt for $\Sigma$ in $\mathrm{LT}^{+}$is as follows: 


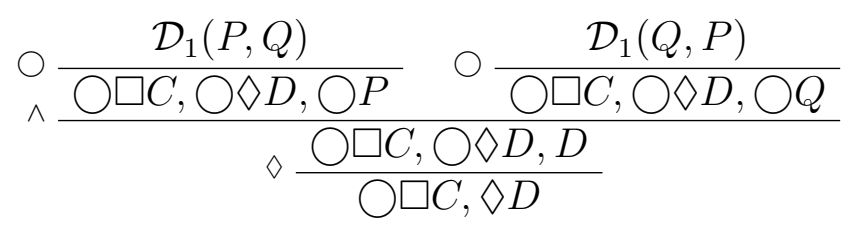

where $\mathcal{D}_{1}(P, Q)$ is

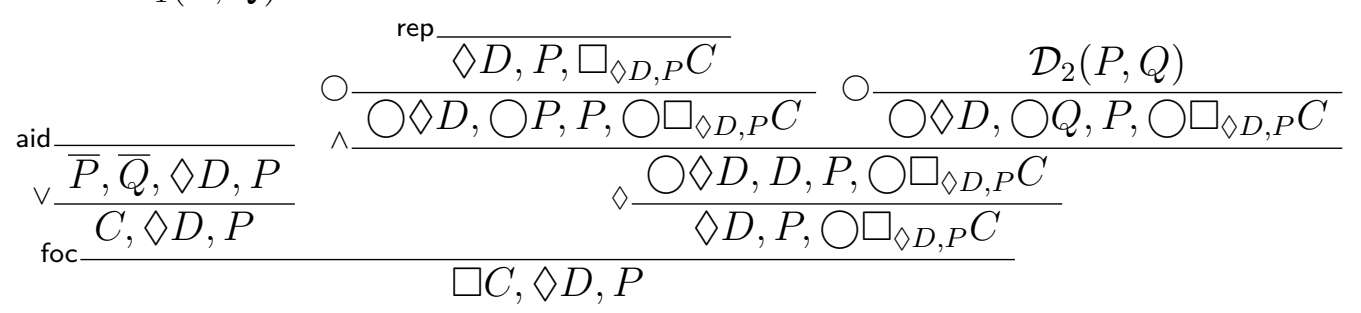

and $\mathcal{D}_{2}(P, Q)$ is

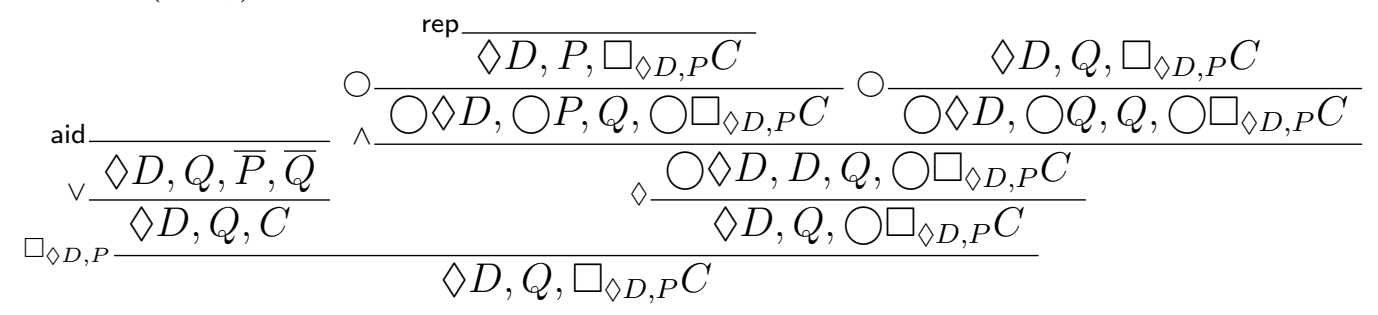

The reason we cannot prove $\Sigma$ in $\mathrm{LT}^{+}$is that it is impossible to prove the sequent $\diamond D, Q, \square_{\diamond D, P} C$ in $\mathrm{LT}^{+}$. Since $\diamond D, Q, \square_{\diamond D, P} C$ is no instance of axiom rep, it is natural to try to prove it by applying the $\square_{\diamond, P}$ rule first. However, as we can see in derivation $\mathcal{D}_{2}(P, Q)$ this leads again to sequent $\diamond D, Q, \square_{\diamond D, P} C$. Hence proof-search for $\Sigma$ fails, i.e. $\mathrm{LT}^{+}{ }^{+}$cannot be complete. However, the fact that an annotated sequent, i.e. $\diamond D, Q, \square_{\diamond D, P} C$, is cyclic in $\mathcal{D}_{2}(P, Q)$ gives us a hint for how we should improve the principle for closing cyclic branches. What if we keep a set of sequents rather than a single sequent in the annotation? This is the approach of Brünnler and Lange [2], which we study in the next section.

\section{Histories}

So far, all our systems could only store one sequent in the annotation. In the previous section we have seen that this not enough to define a complete cutfree sequent system for LTL. Brünnler and Lange [2] present a system that is very similar to LT1. The only difference is that their annotations contain sets of sequents rather than single sequents. Based on their approach, we present a system LT2, for which we syntactically establish weakening. This solves an open problem of Brünnler and Lange. 


\subsection{System LT2}

We start with defining the set of histories (finite sets of sequents), which we call His:

$$
\text { His }=\{H \mid H \text { is a finite subset of Seq }\}
$$

We will use the letters $H$ and $G$ for histories, possibly primed or with subscripts. We also define the set $\mathcal{L}_{\text {his }}$ :

$$
\mathcal{L}_{\text {his }}:=\left\{\square_{H} A, \bigcirc \square_{H} A \mid A \in \mathcal{L}_{\mathcal{S}}, H \in \text { His }\right\}
$$

We will refer to $\mathcal{L}_{\text {his }}$-formulas as formulas with histories, or when there is no danger of confusion as annotated formulas. We also define the set of sequents with histories:

$$
\begin{gathered}
\text { Seq }_{\text {his }}:=\left\{\Gamma \mid \Gamma \text { is a finite subset of } \mathcal{L}_{\mathcal{S}} \cup \mathcal{L}_{\text {his }}\right. \text { that contains } \\
\text { at most one } \left.\mathcal{L}_{\text {his }} \text {-formula }\right\}
\end{gathered}
$$

We assume that a history is semantically equivalent to the conjunction of its elements, i.e. for a model $\mu$ and $i \in \mathbb{N}$ :

$$
\mu, i \models H \quad \Longleftrightarrow \quad \mu, i \models \bigwedge_{\Gamma \in H} \Gamma \quad \Longleftrightarrow \quad \mu, i \models \bigwedge_{\Gamma \in H} \bigvee_{A \in \Gamma} A
$$

The semantics of $\square_{H} A$ is defined like that of $\square_{\Gamma} A$. We have:

$$
\mu, i \models \square_{H} A \quad \Longleftrightarrow \quad \forall j \geq i((\forall i \leq k \leq j(\mu, k \models H)) \Longrightarrow \mu, j \models A)
$$

In Figure 4 we present system LT2. Again we assume $\Sigma \in$ Seq in the $\bigcirc$-rule. For rep, $\square_{H}$ and foc, we also assume $\Gamma \in$ Seq.

Whenever rule $\square_{\Delta}$ is applied in an $\mathrm{LT} 1^{+}$-proof, the sequent in the annotation does not change. In system LT2, an application of rule $\square_{H}$ leads to a new sequent being added to the annotation (history). Thus, rule $\square_{H}$ is a generalization of rule $\square_{\Delta}$, which implies that system $\mathrm{LT}^{+}$is a subsystem of LT2.

System LT2 proves the sequent:

$$
\Sigma=\bigcirc \square(\bar{P} \vee \bar{Q}), \diamond(\bigcirc P \wedge \bigcirc Q)
$$

which as we saw in section 3 cannot be proved in $\mathrm{LT} 1^{+}$. Hence, system $\mathrm{LT} 1^{+}$ is proper subsystem of system LT2. We now present the proof of $\Sigma$ in LT2. As before we set $D=\bigcirc P \wedge \bigcirc Q$ and $C=\bar{P} \vee \bar{Q}$. Moreover, we sometimes write, e.g., $\square_{\Delta, \Gamma}$ for $\square_{\{\Delta, \Gamma\}}$. 


$$
\begin{gathered}
\text { aid } \frac{}{\Gamma, P, \bar{P}} \quad \vee \frac{\Gamma, A, B}{\Gamma, A \vee B} \quad \wedge \frac{\Gamma, A \quad \Gamma, B}{\Gamma, A \wedge B} \\
\text { rep } \frac{\Gamma, \square_{H, \Gamma} A}{\text { foc } \frac{\Gamma, A \quad \Gamma, \bigcirc \square_{\{\Gamma\}} A}{\Gamma, \square A}} \quad \square_{H} \frac{\Gamma, A \quad \Gamma, \bigcirc \square_{H, \Gamma} A}{\Gamma, \square_{H} A} \\
\square \frac{\Gamma, A \quad \Gamma, \bigcirc \square A}{\Gamma, \square A} \quad \diamond \frac{\Gamma, A, \bigcirc \diamond A}{\Gamma, \diamond A} \quad \bigcirc \frac{\Gamma}{\Sigma, \bigcirc \Gamma}
\end{gathered}
$$

Figure 4: System LT2

$$
\curvearrowright \frac{\mathcal{D}_{1}(P, Q)}{\bigcirc \square C, \bigcirc \diamond D, \bigcirc P} \quad \bigcirc \frac{\mathcal{D}_{1}(Q, P)}{\bigcirc \square C, \bigcirc \diamond D, \bigcirc Q}
$$

where $\mathcal{D}_{1}(P, Q)$ is

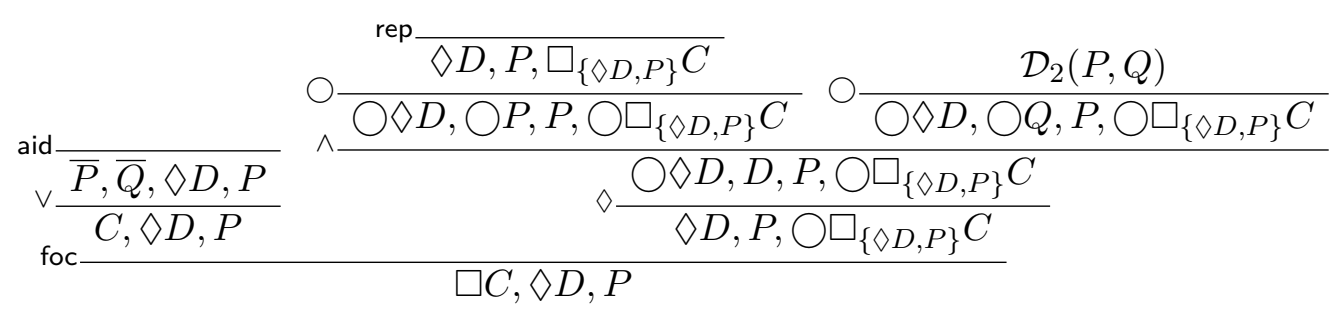

$\mathcal{D}_{2}(P, Q)$ is

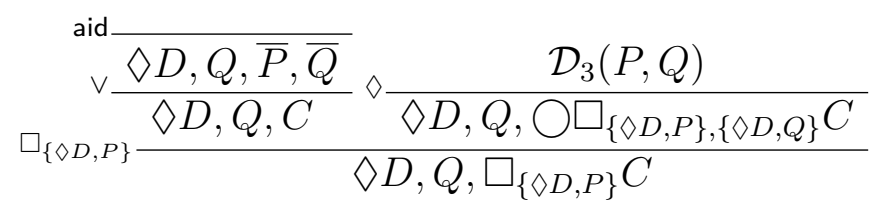

and $\mathcal{D}_{3}(P, Q)$ is

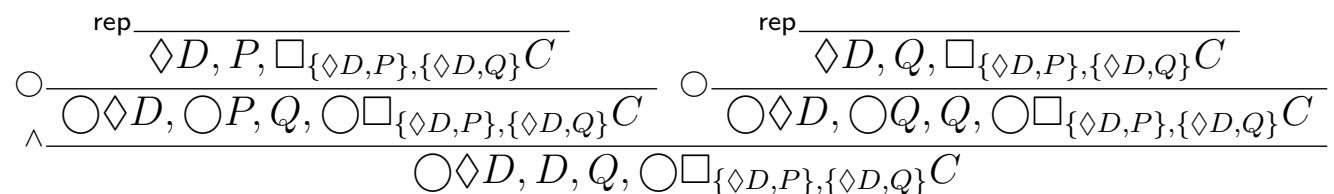

As we mentioned before, Brünnler and Lange's system with histories is very similar to our LT2, so using the same techniques as in [2] we can show soundness and completeness. Actually, we can prove completeness for a system 
$\mathrm{LT}^{\prime}$, in which the $\bigcirc$-rule allows only weakinging with atomic formulas. This system can then be embedded in LT2, thus showing the completeness of LT2.

Theorem 18. System LT2 is sound and complete with respect to LTL-models, i.e. for all $\mathcal{L}_{\mathcal{S}}$-formulas $A$ we have

$$
\models A \quad \Longleftrightarrow \quad \mathrm{LT} 2 \vdash A
$$

\subsection{Weakening}

Brünnler and Lange [2] formulated the problem of syntactically showing the admissibility of weakening in annotated systems. Here we provide a solution to this problem for LT2.

We use the same approach as for LT1. That is we show weakening as a corollary of the next-property. However, the presence of histories in LT2 requires some care. We will need the following two auxiliary statements, which can be shown simultaneously by induction on $n$.

Lemma 19. Let $H$ and $G \in$ His. It holds:

1. If $\mathrm{LT} 2 \vdash^{n} \Gamma, \square_{H} A$, then $\mathrm{LT} 2 \vdash^{n} \Gamma, \square_{H, G} A$.

2. If $\mathrm{LT} 2 \vdash^{n} \Gamma, \bigcirc \square_{H} A$, then $\mathrm{LT} 2 \vdash^{n} \Gamma, \bigcirc \square_{H, G} A$.

The analogue of Lemma 6 for system LT2 is the following lemma:

Lemma 20. Let $\Gamma \in$ Seq. If $\mathrm{LT} 2 \vdash^{n} \Gamma$, then there is an LT2-proof of $\Gamma$ satisfying the next-property.

Proof. Again the proof is by induction on $n$ and a case distinction on the last rule. We only show the case for foc. Then $\Gamma=\Delta, \square A$ and the given proof $\mathcal{E}$ of $\Gamma$ has the following form: 


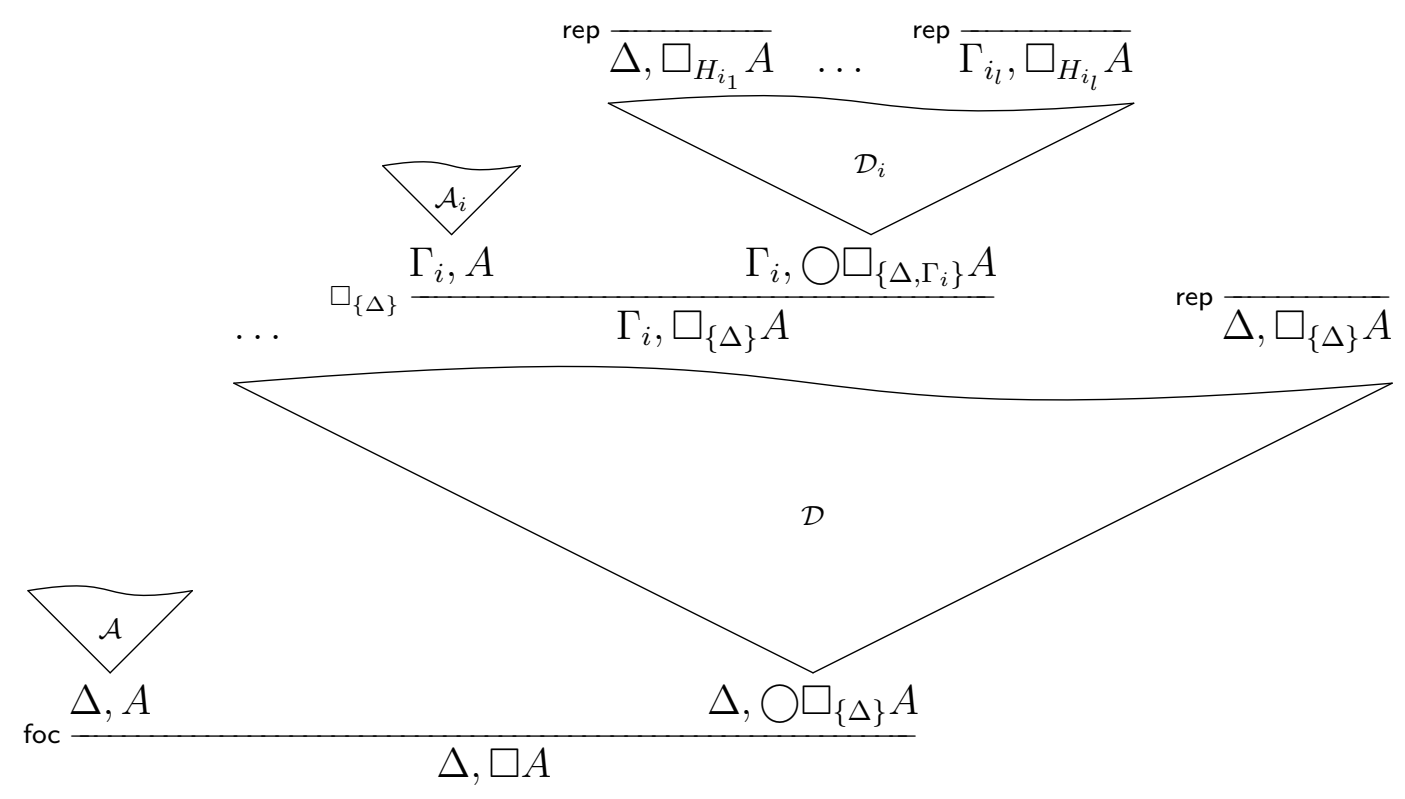

We have that in the derivation $\mathcal{D}$ :

any branch from $\Delta, \bigcirc \square_{\{\Delta\}} A$ to some $\Gamma_{i}, \square_{\{\Delta\}} A$ or some $\Delta, \square_{\{\Delta\}} A$ goes through a $\bigcirc$-rule

and

Furthermore, we observe that if $\Delta \in H_{i_{k}}$ (that is when $\Delta, \square_{H_{i_{k}}} A$ is an instance of rep), then from LT2 $\vdash \Delta, \bigcirc \square_{\{\Delta\}} A$ and Lemma 19 we get a proof $\mathcal{B}_{i_{k}}$ for $\Delta, \bigcirc \square_{H_{i_{k}}} A$.

We let $\mathcal{D}_{i}^{\prime}$ be the the derivation that results from $\mathcal{D}_{i}$ by deleting $\Delta$ from all histories occurring in threads starting from $\bigcirc \square_{\left\{\Delta, \Gamma_{i}\right\}} A$. 
Hence we obtain the following proofs of $\Gamma_{i}, \square A$, which we denote by $\mathcal{C}_{i}$.

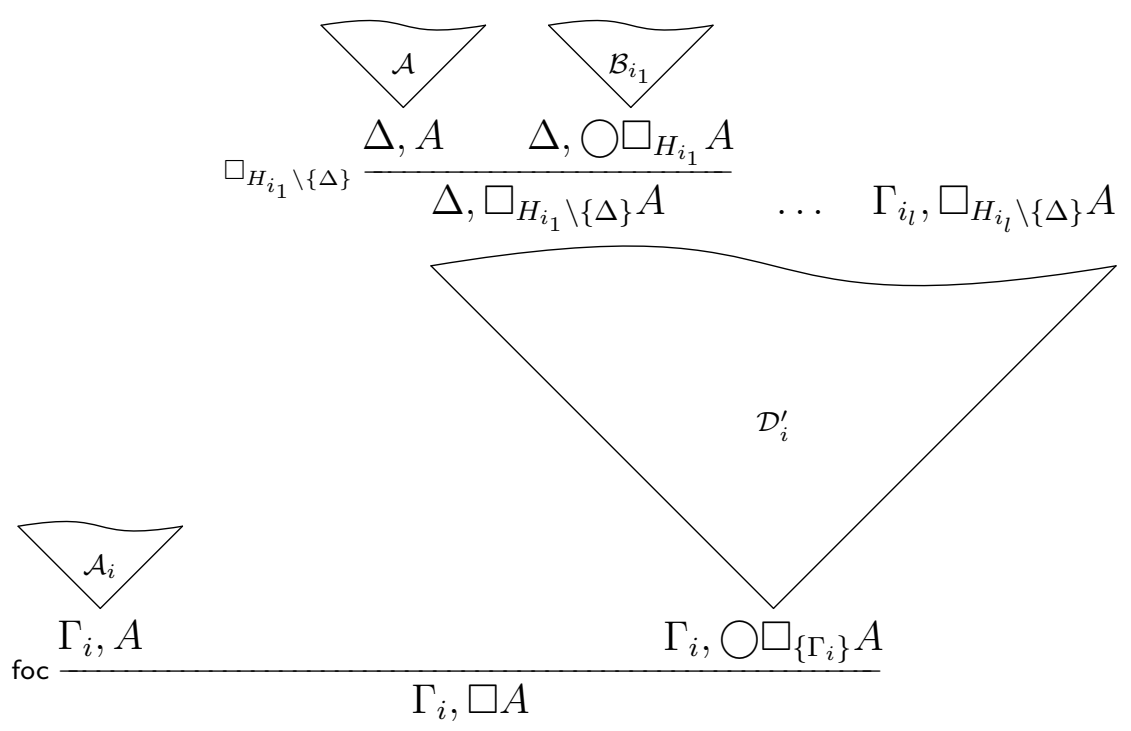

Now we proceed as follows:

1. We apply the induction hypothesis to $\mathcal{A}$, which yields a proof $\mathcal{A}^{\prime}$ of $\Delta, A$ that satisfies the next-property.

2. We let $\mathcal{D}^{\prime}$ be the derivation that results from $\mathcal{D}$ by dropping the annotation $\Delta$ in the threads starting from $\bigcirc \square_{\{\Delta\}} A$.

We find that in the derivation $\mathcal{D}^{\prime}$,

$$
\text { any branch from } \Delta, \bigcirc \square A \text { to some } \Gamma_{i}, \square A \text { or some }
$$

$\Delta, \square A$ goes through a $\bigcirc$-rule

because of (3) and

there are no instances of foc

because of (4). 
Finally we obtain the following proof of $\Delta, \square A$.

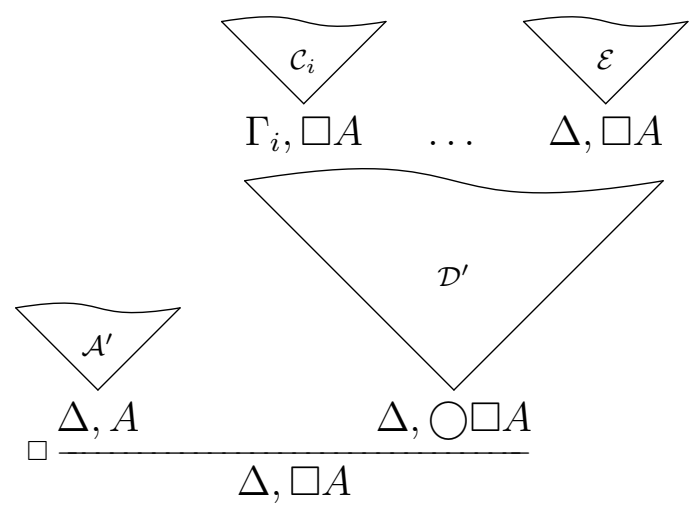

This proof satisfies the next-property. Indeed, we have:

1. the proof $\mathcal{A}^{\prime}$ satisfies the next-property;

2. any branch from $\Delta, \bigcirc \square A$ to some $\Gamma_{i}, \square A$ or to some $\Delta, \square A$ goes through a $\bigcirc$-rule;

3. the derivation $\mathcal{D}^{\prime}$ does not contain instances of foc.

Hence any branch from the conclusion $\Delta, \square A$ to an instance of foc goes through a $\bigcirc$-rule.

We get weakening for LT2 as a corollary of Lemma 20. The proof is the same as for Corollary 7.

Corollary 21 (Weakening for non-annotated sequents). For any $\Gamma, \Delta \in$ Seq we have:

$$
\mathrm{LT} 2 \vdash \Gamma \quad \Longrightarrow \quad \mathrm{LT} 2 \vdash \Gamma, \Delta
$$

\section{Acknowledgements}

We would like to thank the anonymous referee for valuable comments that helped us improve the paper substantially.

\section{References}

[1] J. Brotherston and A. Simpson. Sequent calculi for induction and infinite descent. Journal of Logic and Computation, 21(6):1177-1216, December 2011. 
[2] K. Brünnler and M. Lange. Cut-free sequent systems for temporal logic. The Journal of Logic and Algebraic Programming, 76(2):216-225, 2008.

[3] S. Bucheli, R. Kuznets, and T. Studer. Two ways to common knowledge. In T. Bolander and T. Braüner, editors, Proceedings of the 6th Workshop on Methods for Modalities (M4M-6 2009), Copenhagen, Denmark, 12-14 November 2009, Electronic Notes in Theoretical Computer Science, pages 83-98. Elsevier, 2010.

[4] F. Kröger and S. Merz. Temporal Logic and State Systems. Springer, Berlin, 2008.

[5] M. Lange and C. Stirling. Focus games for satisfiability and completeness of temporal logic. In LICS, 2001.

[6] D. Niwinski and I. Walukiewicz. Games for the mu-calculus. Theoretical Computer Science, 163(1\&2):99-116, 1996.

[7] D. S. Shamkanov. Circular proofs for Gödel-Löb logic. CoRR, abs/1401.4002, 2014.

[8] T. Studer. On the proof theory of the modal mu-calculus. Studia Logica, 89:343-363, 2008.

\section{Addresses}

\section{Ioannis Kokkinis, Thomas Studer}

Institut für Informatik und angewandte Mathematik, Universität Bern Neubrückstrasse 10, 3012 Bern, Switzerland

\{kokkinis,tstuder\}@iam.unibe.ch 